\title{
Association between the serum concentrations and mutational status of IL-8, IL-27 and VEGF and the expression levels of the hERG potassium channel gene in patients with colorectal cancer
}

\author{
MAREWAN N.ABID, FIKRY A. QADIR and ABBAS SALIHI \\ Department of Biology, College of Science, Salahaddin University-Erbil, Erbil, Kurdistan Region 44001, Iraq
}

Received December 16, 2020; Accepted May 21, 2021

DOI: $10.3892 / 01.2021 .12926$

\begin{abstract}
The present study aimed to determine the diagnostic value of the serum levels and mutational status of IL-8, IL-27 and VEGF, and the expression levels of human ether-a-go-go-related gene (hERG) in patients with colorectal cancer (CRC). The serum concentrations were determined using the ELISA technique and genotype variations of IL-8, IL-27 and VEGF were examined using Sanger sequencing, and the expression levels of hERG, which encodes a potassium channel, were determined by quantitative PCR, in blood and tissue samples obtained from 80 patients with CRC and 80 healthy individuals. The results of the present study revealed that the percentage of granulocytes and serum concentrations of carcinoembryonic antigen, IL-8 and IL-27 were significantly increased, whereas the percentage of lymphocytes was decreased in patients with CRC. In total, 31 mutations in three genes (eight mutations in VEGF, 13 mutations in IL-27 and 10 mutations in IL-8) were identified in patients with CRC. The relative mRNA expression levels of hERG were also significantly upregulated in tissue and blood samples of patients with CRC compared with those of healthy individuals. In conclusion, the results of the present study indicated that the increased concentrations and genetic variations of IL-8, IL-27 and VEGF may serve important roles in the development and angiogenic processes of CRC. These changes were concomitant with the upregulation of the expression levels of the potassium channel hERG.
\end{abstract}

\section{Introduction}

According to data published in 2018 by the American Cancer Society, colorectal cancer (CRC) is the second most common type of cancer, with an incidence rate of $10.2 \%$, and the

Correspondence to: Dr Abbas Salihi, Department of Biology, College of Science, Salahaddin University-Erbil, Erbil-Kirkuk Road, Erbil, Kurdistan Region 44001, Iraq

E-mail: abbas.salihi@su.edu.krd

Key words: interleukin-8, interleukin-27, vascular endothelial growth factor, human ether-a-go-go-related gene, colorectal cancer fourth most common cause of cancer-associated mortality worldwide (1), accounting for $\sim 900,000$ deaths per year in 2018 (2,3). In addition to sex, ageing and geographical location, numerous other risk factors have been associated with the increasing incidence of CRC (4). For example, both hereditary and environmental factors are highly associated with the development of CRC (5). According to the mutational origin, CRC can be classified into sporadic, inherited and familial forms (6). Angiogenesis is a complex, tightly regulated process. Several molecules, including VEGF, Notch, angiopoietins and integrins, are known to orchestrate the process (7). Among these factors, VEGF is essential for the induction of physiological and pathological angiogenesis (8). Upregulated expression levels of VEGF have been reported to promote the uncontrolled formation of blood tumor vessels, which has been revealed to be associated with CRC invasiveness, metastasis and poor prognosis (9). Therefore, the majority of current anti-angiogenic treatments for CRC target the VEGF/VEGFR signaling pathway (10). Mutant angiogenic VEGF may provide a genomic basis for the diversity of the tumor-host response and suggests the importance of targeting mutant VEGF with antisense oligonucleotides, since all different VEGF isoforms would have to be neutralized to prevent angiogenesis (11).

IL-8, through binding to its receptors, has been revealed to promote migration, invasion, proliferation and angiogenesis in numerous cancer cell types, including HIF-1 $\alpha$-deficient colon cancer and estrogen receptor-negative breast cancer cells (12). Notably, IL-8 signaling has been demonstrated to promote the malignant progression of tumors and has been associated with inflammatory signaling pathways and gastric cancer (13). SNPs in genes within the IL-8 signaling pathway [IL-8, C-X-C motif chemokine receptor (CXCR)1 and CXCR2] have been reported to be associated with CRC risk (14). The upregulated expression levels of variants of VEGF and IL-8 have been previously revealed to be independently associated with decreased tumor recurrence in patients with stage III CRC, and the analysis of the patients' angiogenic potential based on VEGF and IL-8 genotypes has been suggested to potentially further enhance the efficacy of anti-angiogenic treatment (15). By contrast, IL-27, a member of the IL-12 cytokine family, has demonstrated antitumor activity via stimulation of cytotoxic $\mathrm{T}$ cells and natural killer cell function, regulating the differentiation of $\mathrm{T}$ cell subsets, and suppressing dendritic cell function and angiogenesis (16). In addition, IL-27 gene 
polymorphisms have been revealed to increase the risk of CRC development (17).

The expression levels of hERG, which encodes a potassium channel, have been demonstrated to be highly upregulated in CRC cases with hyperplastic lesions in the colon (18) and in CRC cell lines (19). However, to the best of our knowledge, the association between IL-8, IL-27 and VEGF, and the expression levels of hERG remains unknown. Therefore, the present study aimed to determine the changes in white blood cell (WBC) counts, serum levels of CEA, and the concentrations and mutational status of IL-8, IL-27 and VEGF genes in patients with CRC, and to investigate their association with the expression levels of hERG.

\section{Patients and methods}

Patient samples. The present investigation was based on a case-control study. Study samples were obtained from the Oncology Department of the Hiwa Hospital (Sulaimanya, Kurdistan Region, Iraq) and the Oncology Departments of the Rizgary, Nanakaly and PAR Hospitals (Erbil, Kurdistan Region, Iraq). The present study was approved by the Human Ethics Committee of the College of Science, Salahaddin University-Erbil (N0:4/2/2002; date, 16/6/2019; Erbil, Iraq) and the study was conducted according to the principles of the Declaration of Helsinki. Written informed consent was obtained from all participants for the use of their blood and tissues prior to participation. Venous blood and tissue samples were obtained from 80 patients with CRC (40 male and 40 female patients) and 80 healthy individuals (50 male and 30 female patients). For tissue collection, control samples were obtained from the aforementioned healthy individuals who had negative results following endoscopy and CEA tests. Patients and healthy individuals were recruited between August 2019 and February 2020, and the median age of patients was 55 years (range, 20-70 years), while healthy individuals had a median age of 52 years (range, 23-70 years). Patients with CRC preferably detected by screening and colonoscopy diagnosis were included. The following exclusion criteria were used: i) Patients with underlying immunodeficiency disorders; and ii) immunodeficient individuals who had other co-morbidities that could introduce heterogeneity to the sample, such as additional acquired brain injury, arthritis, chronic obstructive pulmonary disease, asthma, diabetes mellitus, ankylosing spondylitis, connective tissue diseases and other inflammatory diseases.

Tissue and blood collection. All tissue samples were obtained following endoscopy or resection surgery at the PAR Hospital (Erbil, Iraq), while blood samples were collected in a tube containing PBS at the Oncology Department of the Hiwa Hospital (Sulaimanya, Kurdistan Region, Iraq) and the Rizgary Hospital Oncology Department, Nanakaly Hospital, Erbil and PAR Hospital (Erbil, Kurdistan, Iraq), then directly transferred to the Advanced Cancer Biology Laboratory, Department of Biology, College of Science, Salahaddin University-Erbil,Erbil, Iraq for DNA and RNA extraction procedures. Concurrently, blood samples were collected by phlebotomy under the aseptic technique. Blood from both patients and healthy individuals was aspirated into a $5-\mathrm{ml}$ syringe, transferred into a tube and then processed at the Advanced Cancer Biology Laboratory within $1 \mathrm{~h}$. Subsequently, the blood was placed into a clot activator tube for serum preparation. Samples were centrifuged at $1,300 \mathrm{xg}$ for $5 \mathrm{~min}$ in a refrigerated centrifuge at $4^{\circ} \mathrm{C}$ and the separated sera were preserved in an Eppendorf tube, which was stored at $-80^{\circ} \mathrm{C}$ until required for further analysis.

WBC measurements. Total WBC and differential leukocyte counts were determined according to the manufacturer's protocol using an automated Coulter Ac.T diff Hematology analyzer (Beckman Coulter, Inc.).

Estimation of CEA concentration. The concentration of CEA was determined using a Cobas e411 analyzer (Roche Diagnostics) and a ready-to-use reagent kit (Elecsys CEA; Roche Diagnostics) in individual cassettes according to the manufacturer's protocol. This detection method relies on using electrochemiluminescence to measure the immunoreactivity (20).

Determination of $I L-8, I L-27$ and VEGF concentrations. The serum concentrations of IL-8, IL-27 and VEGF were determined using Human Interleukin 8 ELISA Kit (cat. no. E-EL-H6008), Human Interleukin 27 ELISA Kit (cat. no. E-EL-H2338) and Human Vascular Endothelial Growth Factor ELISA Kit (cat. no. E-EL-H1600) (Elabscience, Inc.), respectively, using the sandwich-ELISA method. All reagents and antibodies used for ELISA were included in the kits. The micro-ELISA plates were pre-coated with antibodies specific to IL-8, IL-27 and VEGF. Standards or samples were added to the micro-ELISA plate wells and incubated with the anti-IL-8, anti-IL-27 and anti-VEGF antibodies. A biotinylated detection antibody specific for IL-8, IL-27 or VEGF, avidin-HRP conjugate and substrate were then added to each well. The micro-ELISA plate that contained IL-8, IL-27 and VEGF, biotinylated detection antibodies and the avidin-HRP conjugate appeared blue. The enzyme-substrate reaction was then stopped with $1 \mathrm{M} \mathrm{H}_{2} \mathrm{SO}_{4}$ solution and the color subsequently turned yellow. The absorbance measured spectrophotometrically with an ELISA reader (BioTek Instruments, Inc.) at a wavelength of $450 \mathrm{~nm}$ was proportional to the concentration of IL-8, IL-27 and VEGF. Subsequently, the concentrations in the samples were calculated by comparing the absorbances of the samples to the standard curve.

DNA extraction and quantification. Genomic DNA was purified from CRC tissues using the Tissue Genomic DNA Mini Kit (cat. no. GT050/100/300; Geneaid Biotech Ltd.) according to the manufacturer's protocol. The eluted DNA was assessed for concentration (A260) and purity (A260/A280 ratio) on a Nanodrop spectrophotometer (Thermo Fisher Scientific, Inc.). The Nanodrop instrument was blanked with the elution buffer, and the DNA purity was based on an optimal A260/A280 ratio of 1.8 .

Genotype determination. The present study investigated three commonly studied variants of IL-8, IL-27 and VEGF. Three different locations were selected on the basis of published reports on reference gene expression profiles and previous databases (21-23) as follows: IL-8 [3'-untranslated region (UTR), rs4073], IL-27 (exon region, rs17855750) and VEGF (5'-UTR, rs2010963). 
First, the purified DNA was separately amplified using ready to use master mix (containing Taq DNA polymerase, dNTPs, $\mathrm{MgCl}_{2}$ and reaction buffer; Promega Corporation) for each genetic polymorphism using PCR on a Techne TC-512 gradient thermal cycler (Cole-Parmer,Ltd.) using the following primers: IL-8 rs 4073 forward, 5'-CTAAGAGCAGTAACA GTTCCTAG-3' and reverse, 5'-CATTATGTCAGAGGAAAT TCCACG-3'; IL-27 rs17855750 forward, 5'-GTTCCCTTC CTTCTAAGCT-3' and reverse, 5'-GAAGGTCAGGGAAAC ATCAGG-3'; and VEGF forward, 5'-CTCGGTGCTGGAATT TGATATTC-3' and reverse, 5'-CAAAAGCAGGTCACTCAC TTTGC-3'. The following thermocycling conditions were used for PCR: Initial denaturation at $95^{\circ} \mathrm{C}$ for $5 \mathrm{~min}$; followed by 35 cycles of $95^{\circ} \mathrm{C}$ for $30 \mathrm{sec}$, annealing at $55^{\circ} \mathrm{C}$ for $30 \mathrm{sec}$ and elongation at $72^{\circ} \mathrm{C}$ for $1 \mathrm{~min}$; and a final extension step at $72^{\circ} \mathrm{C}$ for 5 min. All PCR products were separated via $3 \%$ agarose gel electrophoresis, compared with the 50 bp DNA marker (MiniSizer 50 bp DNA Ladder; cat. no 11200; Norgen Biotek Corp.) and stained with safe dye (Safe DNA Gel Stain dye; Add Bio, Inc.) before casting into the tray. Gels were visualized using a gel documentation system (UV Transilluminator UST-20M-8K; Biostep GmbH).

Following the PCR procedure, the product was sent for sequencing to Immunogen Center, Erbil, Iraq (using the same forward primers for each specific region in the gene) on an automatic 3130 genetic analyzer (Applied Biosystems; Thermo Fisher Scientific, Inc.). Sequence data has been included in a curated data repository (GenBank database; https://www.ncbi.nlm.nih.gov/genbank/). Nucleotide sequence data reported are available in the GenBank database under the following accession numbers: MW672394 (www.ncbi.nlm.nih.gov/nuccore/MW672394.1/), MW672395 (www.ncbi.nlm.nih.gov/nuccore/MW672395.1/) and MW672396 (www.ncbi.nlm.nih.gov/nuccore/MW672396.1/). Analysis of the Sanger sequencing data was performed using the Mutation Surveyor software package 5.1 (SoftGenetics, LLC) by comparing with reference genes (AF385628.2, EF064720.1 and AH001553.2).

Reverse transcription- $q P C R$. Total RNA was extracted from blood and tissues using TRIzol ${ }^{\circledR}$ reagent (cat. no. 15596026; Invitrogen; Thermo Fisher Scientific, Inc.). Total RNA was reverse transcribed into cDNA using a RevertAid First Strand cDNA Synthesis Kit (cat. no. K1621; Thermo Fisher Scientific, Inc.). Reverse transcription was performed at $25^{\circ} \mathrm{C}$ for $5 \mathrm{~min}$, followed by incubation for $60 \mathrm{~min}$ at $42^{\circ} \mathrm{C}$. Custom primer was designed to amplify specific regions in the hERG gene and the sequences used were: Forward, 5'-CACCTCCTCGTTGGC ATTG-3' and reverse, 5'-GCTGGCTGTGGTGGACCT-3'. For reverse transcription, in addition to patient's samples, RT-negative, positive control (GAPDH) and no template negative control were setup. qPCR was subsequently performed using a SsoAdvanced Universal SYBR Green Supermix (Bio-Rad Laboratories, Inc.) on a Rotor-Gene ${ }^{\circledR} \mathrm{Q}$ Real-Time PCR cycler (Qiagen $\mathrm{GmbH}$ ). GAPDH served as a reference gene (forward primer, 5'-GAAGGTGAAGGTCGGAGTC-3' and reverse primer, 5'-GAAGATGGTGATGGGATTTC-3'). The relative expression levels were analyzed using the $2^{-\Delta \Delta C q}$ method (24). The qPCR cycling conditions were as follows: 2 min at $95^{\circ} \mathrm{C}$, followed by 40 cycles of denaturation at $95^{\circ} \mathrm{C}$ for $5 \mathrm{sec}$, annealing at $60^{\circ} \mathrm{C}$ for $30 \mathrm{sec}$ and extension at $72^{\circ} \mathrm{C}$ for $45 \mathrm{sec}$, and final extension at $72^{\circ} \mathrm{C}$ for $5 \mathrm{~min}$.

Statistical analysis. Statistical analysis was performed using GraphPad Prism 6.0 software (GraphPad Software, Inc.). Statistical differences in the serological data between patients with CRC $(n=80)$ and healthy individuals $(n=80)$ were determined using a U-Mann Whitney test. Normality tests, namely D'Agostino and Pearson omnibus, Shapiro-Wilk and KS normality tests, were performed for all data. The area under the curve (AUC) for CEA concentration $(n=30)$ was calculated using a receiver operating characteristic curve. The data are presented either as the median and interquartile range or as mean \pm SEM. The strength of the correlation between the expression levels of hERG and the serum concentration of IL-8 and IL-27 was calculated using a nonparametric Spearman correlation test. $\mathrm{P}<0.05$ was considered to indicate a statistically significant difference.

\section{Results}

Total WBC count. The percentage of granulocytes was significantly increased $(\mathrm{P}<0.001)$, whereas the percentage of lymphocytes was significantly decreased $(\mathrm{P}<0.001)$ in patients with CRC compared with in healthy individuals (Fig. 1B and D). However, there were no changes observed in the total WBC count and percentage of monocytes between the patients with CRC and healthy individuals (Fig. 1A and C).

Tumor markers in patients with CRC and healthy individuals. The serum levels of CEA in patients with CRC were significantly increased compared with the levels in healthy individuals $(\mathrm{P}<0.001)$. Furthermore, CEA was revealed to be an efficient biomarker for CRC (AUC, 0.88; Fig. 2).

Serum IL-8, IL-27 and VEGF concentrations. In patients with CRC, the serum concentrations of IL-8 (median, 38.72; range, 28.95-38.72) and IL-27 (median, 35.18; interquartile range, 18.96-49.46) were significantly increased compared with healthy individuals (IL-8 median, 16.96; interquartile range, 13.88-23.11; and IL-27 median, 14.65; interquartile range, 10.17-20.37, respectively; Fig. 3A and B). However, no statistically significant differences were observed in the VEGF concentration between patients with CRC (median, 177.40; interquartile range, 113.00-230.90) and healthy individuals (median, 149.00; interquartile range, 92.66-229.60; Fig. 3C).

Genetic polymorphisms of $I L-8, I L-27$ and VEGF. In total, 31 mutations were identified in the three genes (Table I). In Fig. 4, the rows represent reference, sample and mutation electropherograms, respectively. In VEGF, eight mutations were recorded, 13 mutations were identified in IL-27 and 10 mutations were identified for IL-8. In detail, in VEGF, two types of genomic mutations were recognized: Substitutions $(\mathrm{C}>\mathrm{CG}, \mathrm{A}>\mathrm{AT}, \mathrm{T}>\mathrm{G}$ and $\mathrm{C}>\mathrm{CT}$ ) and deletions (A and TA). The homozygous variant mutation $905 \mathrm{C}>\mathrm{CG} \$ 23$ on chromosome position 6:43738350 has been previously reported in an external public database, dbSNP (https://www.ncbi.nlm.nih. gov/snp/). In the present study, the variant percentage for this mutation was $83.3 \%$. On the other hand, to the best of our 

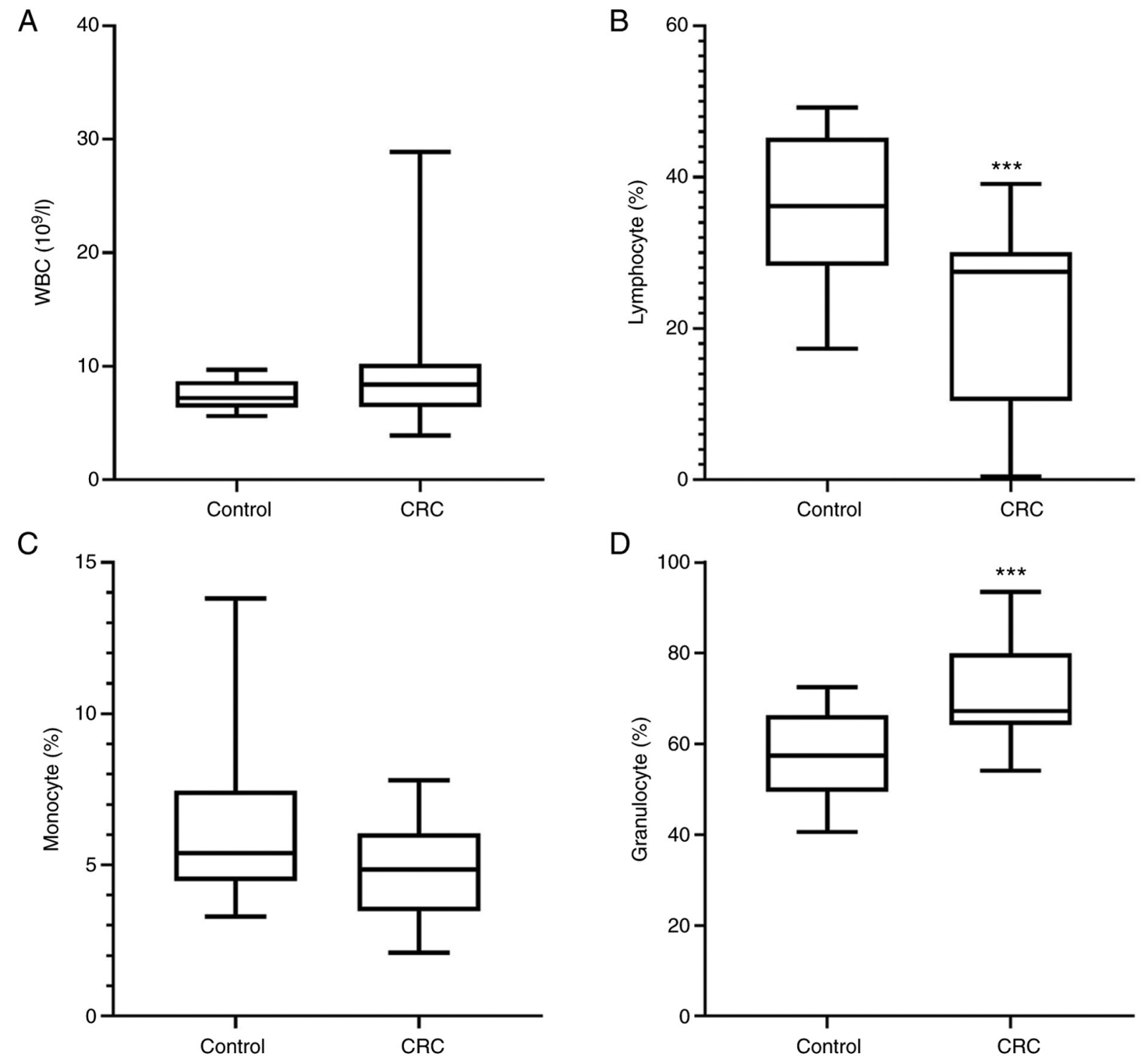

Figure 1. Comparison between total number of WBCs $(10 \% / 1)$, monocyte $(\%)$, lymphocyte $(\%)$ and granulocyte (\%) in a group of healthy individuals and patients with CRC. (A) No significant changes were observed in the total number of WBC. (B) The percentage of lymphocytes was significantly decreased in patients with CRC compared with in individuals in the healthy control group. (C) No significant changes were observed in the percentage of monocytes. (D) The percentage of granulocytes was significantly increased in patients with CRC compared with in individuals in the healthy control group. The comparison was performed using an unpaired Mann-Whitney test. ${ }^{* * *} \mathrm{P}<0.001$ vs. healthy individuals. CRC, colorectal cancer; WBC, white blood cell.
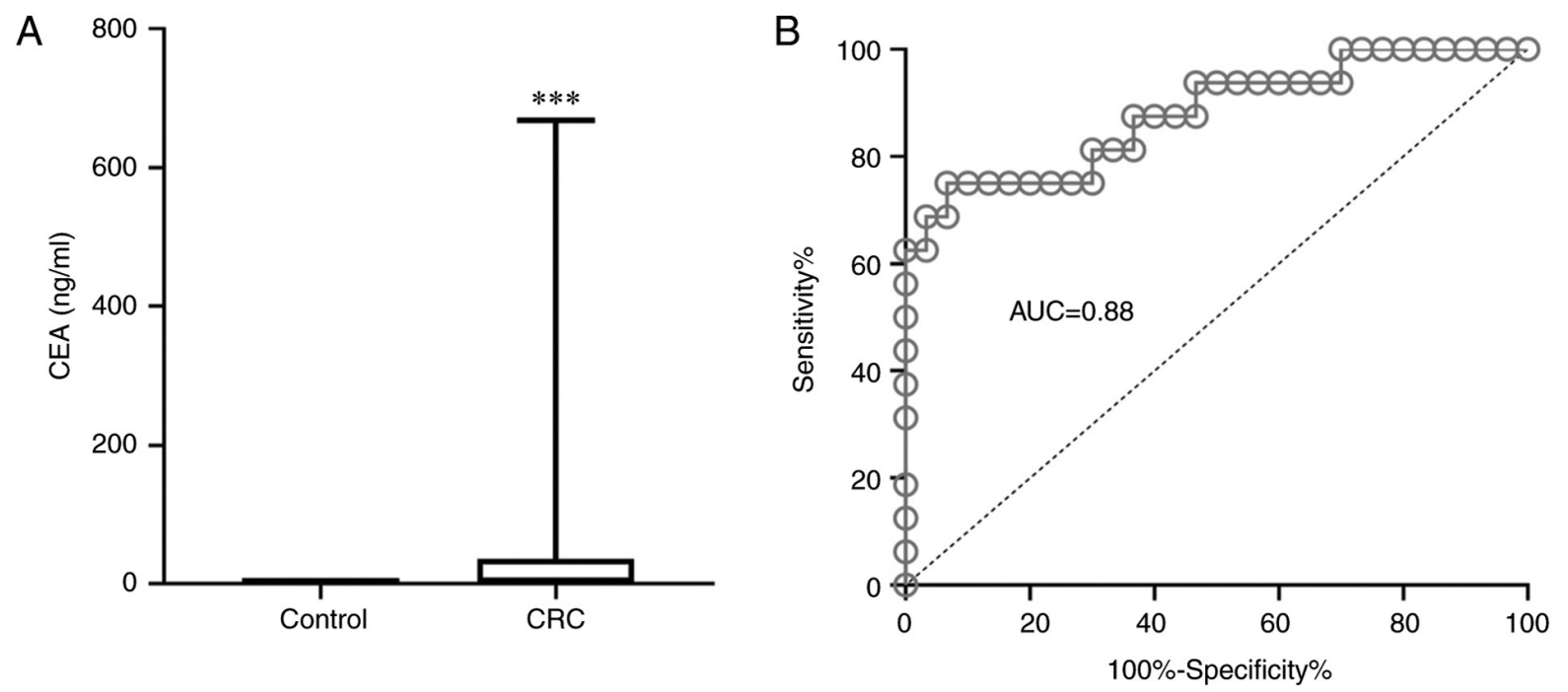

Figure 2. Comparison and AUC value of serum CEA in a group of healthy individuals and patients with CRC. (A) CEA levels were significantly increased in patients with CRC compared with the healthy control group, and the AUC of CEA was 0.88 . The comparison was performed using an unpaired Mann-Whitney test. ${ }^{* * *} \mathrm{P}<0.001$ vs. healthy individuals. (B) While the AUC for CEA concentration was calculated using a receiver operating characteristic curve. AUC, area under the curve; $\mathrm{CRC}$, colorectal cancer. 
Table I. Variants identified in patients with colorectal cancer using Mutation Surveyor software.

\begin{tabular}{|c|c|c|c|c|c|c|c|}
\hline Gene & $\begin{array}{l}\text { Chromosome } \\
\text { position }\end{array}$ & Mutation & $\begin{array}{l}\text { Mutation } \\
\text { genotype }\end{array}$ & $\begin{array}{c}\text { Heterozygous/ } \\
\text { homo zygous }\end{array}$ & Variants & Variant, \% & $\begin{array}{l}\text { External } \\
\text { database }\end{array}$ \\
\hline \multirow[t]{10}{*}{ IL-8 } & 4:74606024 & Substitution & $\mathrm{A}>\mathrm{T}$ & Homozygous & $606 \mathrm{~A}>\mathrm{T} \$ 88$ & 100.0 & dbSNP:4073 \\
\hline & 4:74606126 & Substitution & $\mathrm{T}>\mathrm{TA}$ & Heterozygous & $708 \mathrm{~T}>\mathrm{TA} \$ 10$ & 16.7 & Not found \\
\hline & 4:74605882 & Substitution & $\mathrm{T}>\mathrm{TA}$ & Heterozygous & $464 \mathrm{~T}>\mathrm{TA} \$ 10$ & 50.0 & Not found \\
\hline & $\begin{array}{l}4: 74606163 \\
4: 74606164\end{array}$ & Insertion & $\mathrm{T}$ & Heterozygous & 745_746het_insT\$6 & 16.7 & Not found \\
\hline & 4:74606091 & Duplication & G & Heterozygous & 673het_dupG $\$ 4$ & 16.7 & Not found \\
\hline & 4:74606168 & Substitution & $\mathrm{G}>\mathrm{A}$ & Homozygous & $750 \mathrm{G}>\mathrm{A} \$ 14$ & 33.3 & Not found \\
\hline & 4:74605911 & Substitution & $\mathrm{A}>\mathrm{AT}$ & Heterozygous & $493 \mathrm{~A}>\mathrm{AT} \$ 24$ & 14.3 & Not found \\
\hline & 4:74605919 & Substitution & $\mathrm{A}>\mathrm{AC}$ & Heterozygous & $501 \mathrm{~A}>\mathrm{AC} \$ 24$ & 7.1 & Not found \\
\hline & 4:74606079 & Deletion & $\mathrm{A}$ & Homozygous & 661 delA $\$ 10$ & 8.3 & Not found \\
\hline & 4:74606078 & Substitution & $\mathrm{A}>\mathrm{T}$ & Homozygous & $660 \mathrm{~A}>\mathrm{T}, \$ 16$ & 8.3 & Not found \\
\hline \multirow[t]{14}{*}{ IL-27 } & $16: 28515168$ & Substitution & $\mathrm{G}>\mathrm{GA}$ & Heterozygous & $3488 \mathrm{G}>\mathrm{GA} \$ 42$ & 20.0 & Not found \\
\hline & $16: 28515167$ & Substitution & $\mathrm{A}>\mathrm{AT}$ & Heterozygous & 3489 A $>$ AT $\$ 29$ & 20.0 & Not found \\
\hline & $16: 28515166$ & Substitution & $\mathrm{T}>\mathrm{TA}$ & Heterozygous & $3490 \mathrm{~T}>\mathrm{TA} \$ 50$ & 20.0 & Not found \\
\hline & $16: 28515188$ & Deletion & $\mathrm{C}$ & Homozygous & $3468 \mathrm{delC} \$ 10$ & 8.3 & Not found \\
\hline & $16: 28515185$ & Deletion & $\mathrm{A}$ & Homozygous & 3471delA $\$ 11$ & 9.1 & Not found \\
\hline & $16: 28515163$ & Deletion & G & Heterozygous & 3493het_delG $\$ 10$ & 16.7 & Not found \\
\hline & $16: 28515270$ & Substitution & $\mathrm{A}>\mathrm{AG}$ & Heterozygous & $3386 \mathrm{~A}>\mathrm{AG}, 45 \mathrm{R}>\mathrm{R} / \mathrm{G} \$ 17$ & 5.6 & Not found \\
\hline & $16: 28515322$ & Insertion & $\mathrm{K}$ & Homozygous & 3333_3334insK $\$ 11$ & 5.6 & Not found \\
\hline & $16: 28515323$ & & & & & & \\
\hline & $16: 28515320$ & Substitution & $\mathrm{G}>\mathrm{C}$ & Homozygous & $3336 \mathrm{G}>\mathrm{C}, 28 \mathrm{G}>\mathrm{G} \$ 8$ & 5.6 & Not found \\
\hline & $16: 28515319$ & Substitution & $\mathrm{A}>\mathrm{AT}$ & Heterozygous & $3337 \mathrm{~A}>\mathrm{AT}, 28 \mathrm{G}>\mathrm{G} / \mathrm{G} \$ 31$ & 16.7 & Not found \\
\hline & $16: 28515349$ & Substitution & $\mathrm{C}>\mathrm{CT}$ & Heterozygous & $3307 \mathrm{C}>\mathrm{CT}, 18 \mathrm{P}>\mathrm{P} / \mathrm{P} \$ 30$ & 5.6 & Not found \\
\hline & $\begin{array}{l}16: 28515214 \\
16: 28515215\end{array}$ & Deletion & $\mathrm{GC}$ & Heterozygous & 3441_3442delGC $\$ 10$ & 6.2 & Not found \\
\hline & $16: 28515195$ & Deletion & $\mathrm{A}$ & Heterozygous & 3461het_delA,\$5 & 7.7 & Not found \\
\hline \multirow[t]{8}{*}{ VEGF } & $6: 43738350$ & Substitution & $\mathrm{C}>\mathrm{CG}$ & Heterozygous & $905 \mathrm{C}>\mathrm{CG} \$ 23$ & 83.3 & dbSNP:2010963 \\
\hline & $\begin{array}{l}6: 43738136 \\
6: 43738137\end{array}$ & Deletion & $\mathrm{TA}$ & Heterozygous & 691_692delTA $\$ 12$ & 20.0 & Not found \\
\hline & 6:43738145 & Substitution & $\mathrm{A}>\mathrm{AT}$ & Heterozygous & $700 A>A T \$ 26$ & 20.0 & Not found \\
\hline & $6: 43738147$ & Substitution & $\mathrm{T}>\mathrm{G}$ & Homozygous & $702 \mathrm{~T}>\mathrm{G} \$ 9$ & 16.7 & Not found \\
\hline & $6: 43738165$ & Substitution & $\mathrm{A}>\mathrm{AT}$ & Heterozygous & $720 \mathrm{~A}>\mathrm{AT} \$ 21$ & 16.7 & Not found \\
\hline & $6: 43738200$ & Deletion & $\mathrm{A}$ & Heterozygous & 755het_delA\$4 & 12.5 & Not found \\
\hline & $6: 43738161$ & Deletion & $\mathrm{A}$ & Homozygous & 716delA $\$ 11$ & 16.7 & Not found \\
\hline & $6: 43738204$ & Substitution & $\mathrm{C}>\mathrm{CT}$ & Heterozygous & $759 \mathrm{C}>\mathrm{CT} \$ 17$ & 14.3 & Not found \\
\hline
\end{tabular}

knowledge, the remaining mutational variants of VEGF have not been reported in external databases.

With regards to IL-27, the present data revealed two distinctive features. First, IL-27 exhibited the highest rate of mutations among the three genes, and second, to the best of our knowledge, none of the mutations have been previously reported in an external database. The analysis of the sequencing data of IL-27 revealed three types of mutation: Substitution (G>GA, A>AT, T>TA, A>AG, G>C and $\mathrm{C}>\mathrm{CT}$ ), deletions (C, A, G, GC and $\mathrm{A}$ ) and insertions $(\mathrm{K})$, and both homozygous and heterozygous variant mutations were identified. The heterozygous variant deletions, 3493het_delG $\$ 10$ and 3461het_delA, \$5, were observed in chromosome positions 16:28515163 and 16:28515195, respectively.
The analysis of the sequencing data of IL-8 revealed four different types of genomic mutations: Substitutions $(A>T$, $\mathrm{T}>\mathrm{TA}, \mathrm{G}>\mathrm{A}, \mathrm{A}>\mathrm{AT}$ and $\mathrm{A}>\mathrm{AC}$ ), insertions ( $\mathrm{T})$, deletions (A) and duplications $(\mathrm{G})$. The homozygous variant mutation $606 \mathrm{~A}>\mathrm{T} \$ 88$ on chromosome position 4:74606024 has been previously reported in external public databases, and its variant percentage in the present study was $100 \%$. The heterozygous insertion 745_746het_insT\$6 and the heterozygous duplication 673het_dupG $\$ 4$ were identified on chromosome positions 4:74606163_4:74606164 and 4:74606091, respectively.

Expression levels of $h E R G$ and correlation with $I L-8$ and $I L-27$. The relative mRNA expression levels of hERG were significantly upregulated in the tissue $(66.4 \pm 27.1)$ and blood 

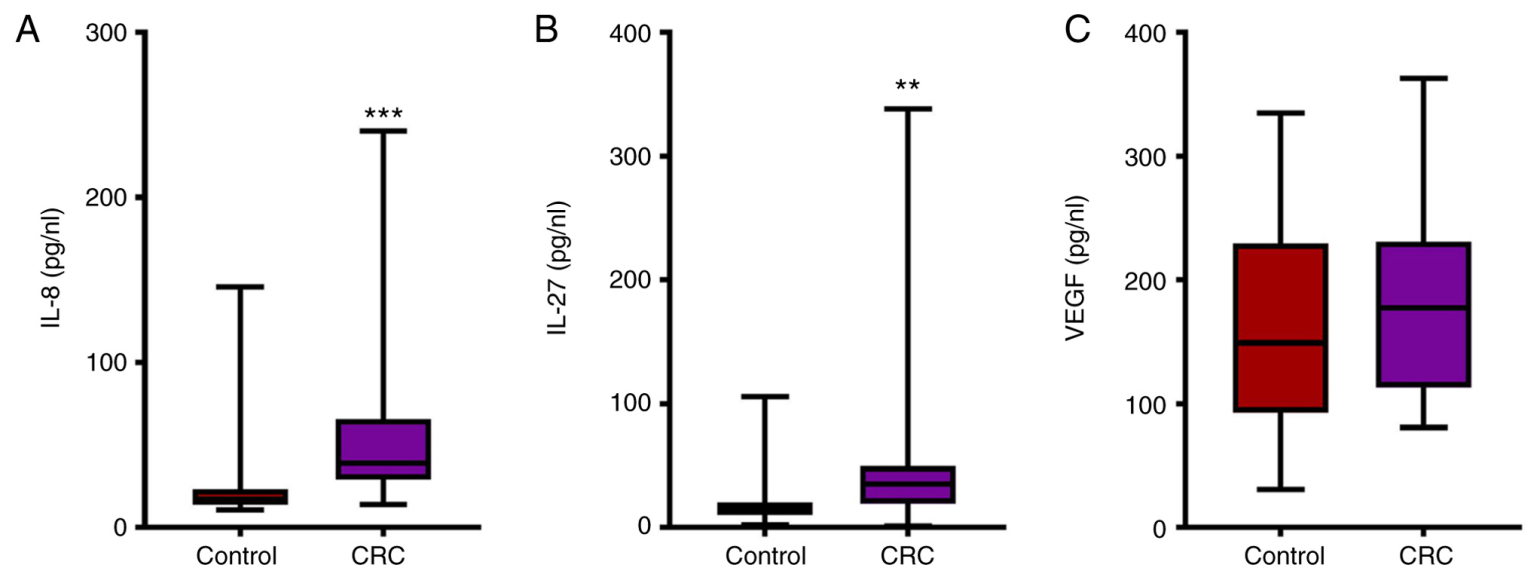

Figure 3. Comparison between IL-8 (pg/nl), IL-27 (pg/nl) and VEGF (pg/nl) levels in a group of healthy individuals and patients with CRC. (A) IL-8 and (B) IL-27 levels were significantly increased in patients with CRC compared with the healthy control group, (C) while no significant changes were observed in the VEGF levels. The comparison was performed using an unpaired Mann-Whitney test. ${ }^{* * *} \mathrm{P}<0.01$ and ${ }^{* * *} \mathrm{P}<0.001$ vs. healthy individuals. CRC, colorectal cancer.

A

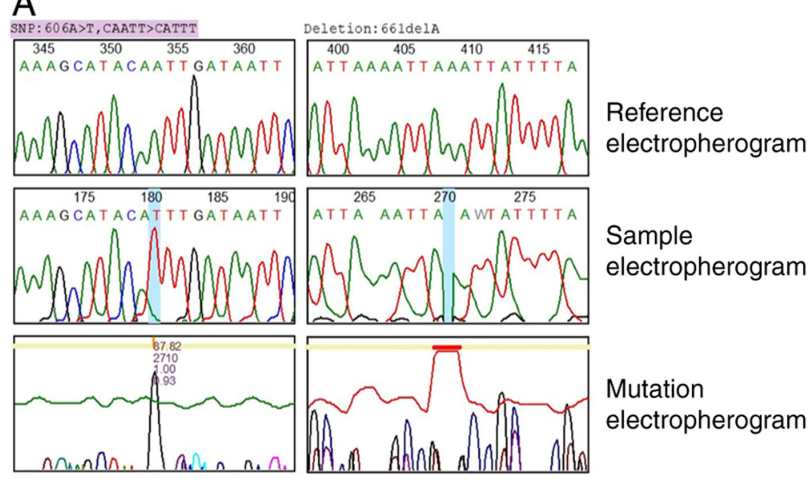

B

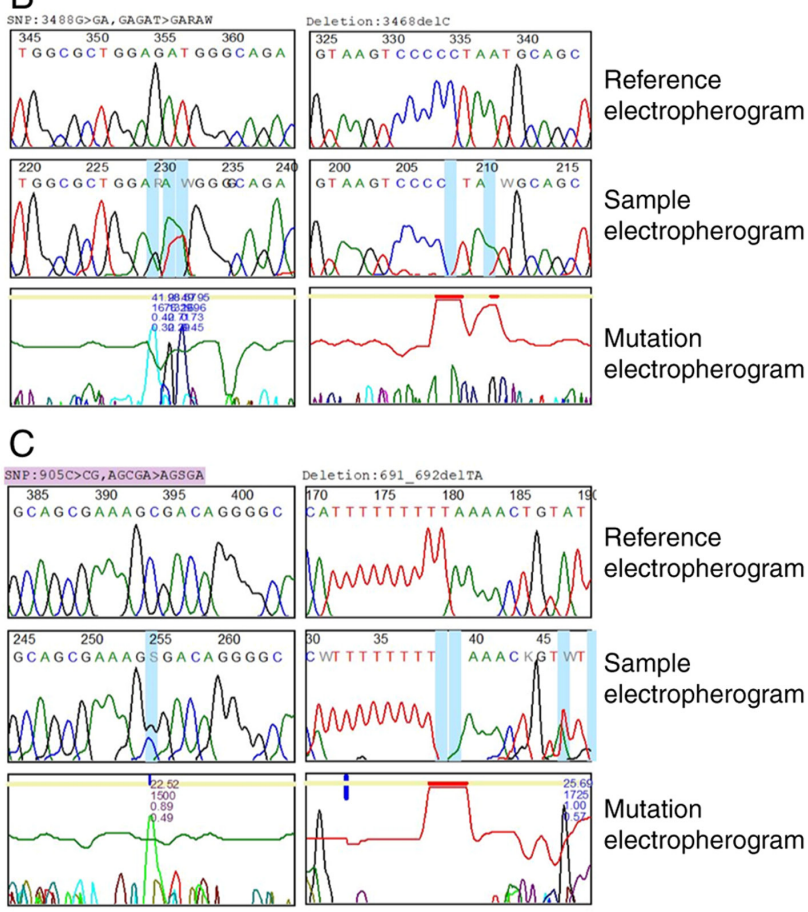

Figure 4. DNA sequence electropherograms showing nucleotide mutations in IL-8, IL-27 and VEGF. (A) Homozygous substitution from A to T and homozygous deletion 661A in 3'-UTR, rs4073 of IL-8 gene. (B) Heterozygous substitution from $\mathrm{G}$ to $\mathrm{GA}$ and homozygous deletion $3468 \mathrm{C}$ in exon region, rs17855750 of IL-27 gene. (C) Heterozygous substitution from C to CG and heterozygous deletion 691_692TA in 5'-UTR, rs2010963 of VEGF gene. UTR, untranslated region.
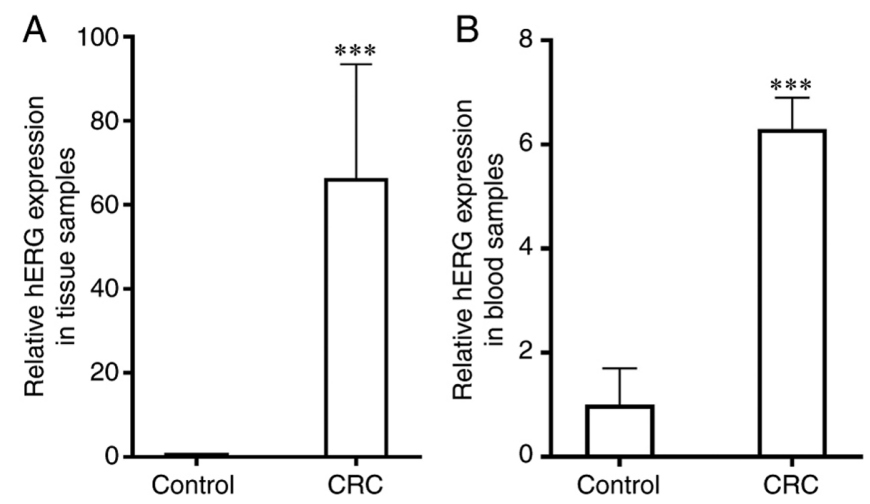

Figure 5. Relative fold expression of hERG channel in a group of healthy individuals and patients with CRC. The relative fold expression was significantly increased in (A) tissue samples and (B) blood samples of patients with CRC compared with the healthy control group. The comparison was performed using an unpaired Mann-Whitney test. ${ }^{* * *} \mathrm{P}<0.001$ vs. healthy individuals. CRC, colorectal cancer; hERG, human ether-a-go-go-related gene.

samples $(6.3 \pm 0.6)$ from patients with CRC compared with healthy individuals $(1.0 \pm 0.0$ and $1.0 \pm 0.7$, respectively; $\mathrm{P}<0.001$; Fig. 5). A positive non-significant correlation was identified between the expression levels of hERG and IL-8 $(\mathrm{r}=0.43 ; \mathrm{P}=0.42)$ and IL-27 ( $\mathrm{r}=0.029 ; \mathrm{P}=0.99$; Fig. 6).

\section{Discussion}

To the best of our knowledge, a link has been observed between serum WBC count and CEA levels in patients with CRC (25). This association could be used as a predictive factor not only in CRC but also in other types of cancer. It is crucial to identify predictive and prognostic factors in tumor development because this could help in finding the most effective treatments. The findings of this investigation matched those of Tsai et al (26), who found that certain WBC cells, notably the neutrophil-lymphocyte ratio (NLR) and CEA levels, could be prognostic and predictive markers for advanced-stage CRC. Increased expression of neutrophils and lymphocytes is associated with systemic inflammation and a signal of impaired cell-mediated immunity, respectively, according to the best 

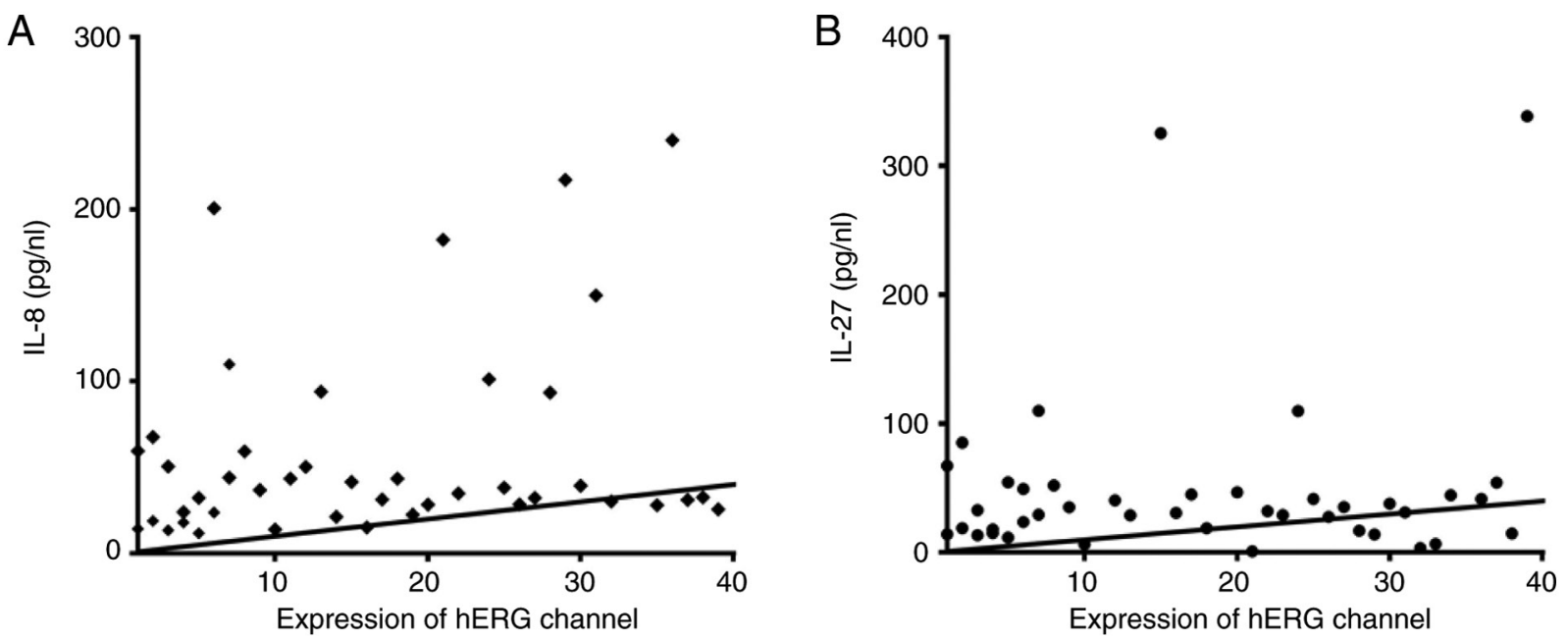

Figure 6. Correlation between (A) IL-8 concentrations, (B) IL-27 and the relative expression levels of hERG channel. A positive non-significant correlation was identified between the expression levels of hERG and IL-8 ( $r=0.43 ; \mathrm{P}=0.42)$ and IL-27 ( $r=0.029 ; \mathrm{P}=0.99)$. hERG, human ether-a-go-go-related gene.

explanation for their relationship (27). NLR expression is high, which inhibits lymphokine-activated killer cells (28). As a result, it may enhance the risk of tumor metastasis in patients with CRC (29). The presence of more inflammatory cells in the tumor microenvironment may explain why these cells, particularly neutrophils, act as an angiogenic activator in metastatic CRC (30). According to a previous study, elevated CEA levels in the blood are linked to a poor prognosis, a decreased survival rate, and advanced metastatic stage in cancer cells (31). Lee et al (32) demonstrated that a high NLR was mainly observed in an advanced stage of tumor progression, which suggested its potential use as a predictor of the incidence and mortality of CRC. Increased levels of WBC are known to enhance the levels of inflammation, which increases the likelihood of tumor metastasis (33). The levels of CEA have been reported to be elevated in metastatic tumors (34). Therefore, the association between CEA levels and the NLR may be a valuable predictive factor to monitor metastatic CRC, which could be extended for use in other types of cancer.

The levels of IL-8, a proinflammatory cytokine, which has angiogenic activity, are reportedly increased in numerous types of tumor, including CRC (12). In CRC, IL-8 has been revealed to act on cancer cells via their receptors to promote migration, invasion and proliferation, in addition to angiogenesis in vivo (12). In the present study, the concentration of IL-8 was significantly increased in patients with CRC compared with in healthy individuals. The increase in the concentration of IL- 8 has been previously reported in CRC tissues and cell lines (35); the results revealed that the concentrations of IL-8 were increased in CRC tissues compared with healthy colon tissues. SNPs of driver genes have been reported to serve a role in the development of multiple neoplasms, including CRC (36). The sequencing results in the present study identified several SNPs in the IL-8 gene in the CRC tissues: Seven mutations, one insertion, one deletion and one duplication mutation. Bondurant et al (14) reported that the SNP, rs4073, in IL-8 is associated with an increased CRC risk. Furthermore, another previous study involving Malaysian patients with CRC revealed that the homozygous variant of IL-8-251AA was associated with a higher susceptibility of CRC compared with the homozygous wild-type-251TT genotype (37). The IL-8-251T >A polymorphism has been reported to be associated with a higher promoter activity, which in turn increases the levels of IL- 8 and promotes the proangiogenic effects of IL-8, and ultimately increases tumor cell proliferation (38).

In the present study, the levels of IL-27 were increased in patients with CRC compared with in healthy individuals. These findings were not consistent with previous studies, which reported that IL-27 served as a tumor suppressor in several types of cancer, including B16F10 melanoma and Lewis lung carcinoma $(39,40)$. Data collected from DNA sequencing in the present study revealed multiple mutations in three different exon regions in the IL-27 gene. These results are controversial; the levels of IL-27 were increased in patients with CRC, whereas several genetic mutations, including SNPs, insertions and deletions were observed in several regions of the IL-27 gene. In normal cell environments, IL-27 acts as a tumor suppressive and anti-angiogenic factor (41). However, based on the DNA sequencing data in the present study, IL-27 was revealed to be mutated in the tumor microenvironment of patients with CRC. These findings suggested that IL-27 may lose its tumor suppressive role, which also provides an explanation for the high serum levels of IL-27 in the patients with CRC. A previous study revealed that the IL-27 polymorphism is associated with a high risk of CRC (17).

IL-27 exerts a tumor-suppressive role in several types of cancer cells by inhibiting tumor cell proliferation, angiogenesis, invasion and survival, in addition to activating antitumor immune responses (42). However, several previous studies have reported that IL-27 serves a protumorigenic role (41-43). Diakowska et al (44) reported that the serum levels of IL-27 are increased in gastrointestinal tract cancer, which is associated with a higher lymph node status. Similarly, Lu et al (45) revealed that in patients with breast cancer, elevated serum IL-27 levels are associated with VEGF expression levels and clinical stage. A similar inhibitory role of IL-27 has been reported in prostate cancer cells, in which high levels of IL-27 are associated with the inhibition of prostate cancer cell survival and proliferation (46). These findings, combined with the DNA sequencing findings of the present study, suggested 
that the protumorigenic role of IL-27 may be another possible explanation for the high serum levels of IL-27 in patients with CRC. Therefore, CRC may be used as an example to demonstrate the dual role of IL-27 in normal and tumor cells.

Among several angiogenic factors, VEGF is an essential factor in mCRC (47). In the present study, DNA sequencing data revealed several DNA abnormalities, including SNPs, and insertion and deletion mutations in three different exon regions of the VEGF gene. The results of ELISA revealed that the levels of VEGF in patients with CRC were not statistically significantly different compared with the controls. These results were not consistent with the findings of a number of previous studies, which reported that VEGF may be a crucial factor for inducing the proliferation of endothelial cells and its expression levels are upregulated in the majority of patients with mCRC $(48,49)$. On the other hand, Jubb et al (50) reported that the levels of VEGF in patients with CRC following bevacizumab treatment were downregulated compared with the control individuals. The explanation for the changes in the expression levels of VEGF not being statistically significant in patients with $\mathrm{mCRC}$ in the present study may be due to the fact that the majority of the patients with $\mathrm{mCRC}$ were undergoing chemotherapy. Bevacizumab can directly interact with VEGF and block its biological activity, which may eventually inhibit its crucial role as a proangiogenic factor in CRC development (47).

In the present study, upregulation of hERG channel expression could be a suitable biomarker for detecting patients with CRC, since hERG channels are frequently upregulated in solid cancer types and such expression is associated with clinicopathological features (18). An important role of the hERG channel in CRC carcinogenesis in vivo is that early upregulation of the expression of the hERG gene marks precancerous lesions that undergo malignant progression (51). Since there has been no previous study linking the expression levels of the hERG channel to IL-8, IL-27 or VEGF in patients with CRC, the present study was the first to link the expression levels of the hERG channel to IL-8, IL-27 or VEGF in patients with CRC. This holds great promise for future pharmacological targeted CRC therapy. A limitation of the present study was that only the serum concentrations of IL-8 and IL-27 was measured. Therefore, future studies should focus on assessing the molecular signaling pathways in CRC tissues and cell lines to provide a more comprehensive association between hERG expression and interleukin signaling.

In conclusion, the findings of the present study indicated that the genetic variations of IL-8, IL-27 and VEGF might serve important roles in the development and angiogenesis of $\mathrm{CRC}$. These changes were suggested to be associated with the upregulated expression levels of the hERG channel gene. These data may enhance the current understanding of the molecular mechanisms of CRC angiogenesis and may help determine a novel target for the treatment of CRC.

\section{Acknowledgements}

Not applicable.

\section{Funding}

No funding was received.

\section{Availability of data and materials}

The datasets used and/or analyzed during the current study are available from the corresponding author on reasonable request.

\section{Authors' contributions}

MNA and FAQ performed experiments. MNA and AS designed the experiments, analyzed data and co-wrote the manuscript. MNA, FAQ and AS confirm the authenticity of all the raw data. All authors read and approved the final manuscript.

\section{Ethics approval and consent to participate}

The present study was authorized and approved by the Human Ethics Committee of Salahaddin University-Erbil, Erbil, Iraq. Patients provided informed written informed consent.

\section{Patient consent for publication}

All patients provided written informed consent for the publication of data in the present study.

\section{Competing interests}

The authors declare that they have no competing interests.

\section{References}

1. Wen J, Min X, Shen M, Hua Q, Han Y, Zhao L, Liu L, Huang G, Liu J and Zhao X: ACLY facilitates colon cancer cell metastasis by CTNNB1. J Exp Clin Cancer Res 38: 401, 2019.

2. Hirata A, Hatano Y, Niwa M, Hara A and Tomita H: Heterogeneity of colon cancer stem cells. Adv Exp Med Biol 1139: 115-126, 2019.

3. Rawla P, Sunkara T and Barsouk A: Epidemiology of colorectal cancer: Incidence, mortality, survival, and risk factors. Prz Gastroenterol 14: 89-103, 2019.

4. Wong MC, Ding H, Wang J, Chan PS and Huang J: Prevalence and risk factors of colorectal cancer in Asia. Intest Res 17: 317-329, 2019.

5. Thanikachalam $\mathrm{K}$ and Khan G: Colorectal cancer and nutrition. Nutrients 11: 164, 2019.

6. Fearon ER and Vogelstein B: A genetic model for colorectal tumorigenesis. Cell 61: 759-767, 1990.

7. Sun W: Angiogenesis in metastatic colorectal cancer and the benefits of targeted therapy. J Hematol Oncol 5: 63, 2012.

8. Lan J, Li H, Luo X, Hu J and Wang G: BRG1 promotes VEGF-A expression and angiogenesis in human colorectal cancer cells. Exp Cell Res 360: 236-242, 2017.

9. Ceci C, Atzori MG, Lacal PM and Graziani G: Role of VEGFs/VEGFR-1 signaling and its inhibition in modulating tumor invasion: Experimental evidence in different metastatic cancer models. Int J Mol Sci 21: 1388, 2020.

10. Deng F, Zhou R, Lin C, Yang S, Wang H, Li W, Zheng K, Lin W, Li X, Yao X, et al: Tumor-secreted dickkopf 2 accelerates aerobic glycolysis and promotes angiogenesis in colorectal cancer. Theranostics 9: 1001-1014, 2019.

11. Uthoff SM, Duchrow M, Schmidt MHH, Broll R, Bruch HP, Strik MW and Galandiuk S: VEGF isoforms and mutations in human colorectal cancer. Int J Cancer 101: 32-36, 2002.

12. Ning Y and Lenz HJ: Targeting IL-8 in colorectal cancer. Expert Opin Ther Targets 16: 491-497, 2012.

13. Waugh DJ and Wilson C: The interleukin-8 pathway in cancer. Clin Cancer Res 14: 6735-6741, 2008.

14. Bondurant KL, Lundgreen A, Herrick JS, Kadlubar S, Wolff RK and Slattery ML: Interleukin genes and associations with colon and rectal cancer risk and overall survival. Int J Cancer 132: 905-915, 2013. 
15. Lurje G, Zhang W, Schultheis AM, Yang D, Groshen S, Hendifar AE, Husain H, Gordon MA, Nagashima F, Chang HM and Lenz HJ: Polymorphisms in VEGF and IL-8 predict tumor recurrence in stage III colon cancer. Ann Oncol 19: 1734-1741, 2008.

16. Liang Y, Chen Q, Du W, Chen C, Li F, Yang J, Peng J, Kang D, Lin B, Chai X, et al: Epstein-barr virus-induced gene 3 (EBI3) blocking leads to induce antitumor cytotoxic T lymphocyte response and suppress tumor growth in colorectal cancer by bidirectional reciprocal-regulation STAT3 signaling pathway. Mediators Inflamm 2016: 3214105, 2016.

17. Lyu S, Ye L, Wang O, Huang G, Yang F, Liu Y and Dong S: IL-27 rs153109 polymorphism increases the risk of colorectal cancer in Chinese Han population. Onco Targets Ther 8: 1493-1497, 2015.

18. Lastraioli E, Lottini T, Bencini L, Bernini M and Arcangeli A hERG1 potassium channels: Novel biomarkers in human solid cancers. Biomed Res Int 2015: 896432, 2015.

19. Crociani O,ZanieriF,Pillozzi S,Lastraioli E,Stefanini M,Fiore A Fortunato A, D'Amico M, Masselli M, De Lorenzo E, et al: hERG1 channels modulate integrin signaling to trigger angiogenesis and tumor progression in colorectal cancer. Sci Rep 3 . $3308,2013$.

20. Wei PL, Lee LT, Tseng LM and Huang KW: Validation of assaying carcinoembryonic antigen in human serum by using immunomagnetic reduction. Sci Rep 8: 10002, 2018.

21. Vislovukh A, Vargas TR, Polesskaya A and Groisman I: Role of 3'-untranslated region translational control in cancer development, diagnostics and treatment. World J Biol Chem 5: 40-57, 2014.

22. Zhang YF and Zhao AD: Common polymorphisms in IL-27 genes may contribute to risk of various human diseases in Asian populations: A meta-analysis. Med Sci Monit 22: 766-775, 2016.

23. Jain L, Vargo CA, Danesi R, Sissung TM, Price DK, Venzon D, Venitz J and Figg WD: The role of vascular endothelial growth factor SNPs as predictive and prognostic markers for major solid tumors. Mol Cancer Ther 8: 2496-2508, 2009.

24. Livak KJ and Schmittgen TD: Analysis of relative gene expression data using real-time quantitative PCR and the 2(-Delta Delta C(T)) method. Methods 25: 402-408, 2001

25. Kang HY, Choe EK, Park KJ and Lee Y: Factors requiring adjustment in the interpretation of serum carcinoembryonic antigen: A cross-sectional study of 18,131 healthy nonsmokers Gastroenterol Res Pract 2017: 9858931, 2017.

26. Tsai PL, Su WJ, Leung WH, Lai CT and Liu CK Neutrophil-lymphocyte ratio and CEA level as prognostic and predictive factors in colorectal cancer: A systematic review and meta-analysis. J Cancer Res Ther 12: 582-589, 2016.

27. Chen L, Deng H, Cui H, Fang J, Zuo Z, Deng J, Li Y, Wang X and Zhao L: Inflammatory responses and inflammation-associated diseases in organs. Oncotarget 9: 7204-7218, 2017.

28. Mogensen TH: Pathogen recognition and inflammatory signaling in innate immune defenses. Clin Microbiol Rev 22: 240-273, 2009.

29. Xu W, He Y, Wang Y, Li X, Young J, Ioannidis JPA, Dunlop MG and Theodoratou E: Risk factors and risk prediction models for colorectal cancer metastasis and recurrence: An umbrella review of systematic reviews and meta-analyses of observational studies. BMC Med 18: 172, 2020.

30. Singel KL and Segal BH: Neutrophils in the tumor microenvironment: Trying to heal the wound that cannot heal. Immuno Rev 273: 329-343, 2016

31. Yan C, Hu Y, Zhang B, Huang K, Zhao H, Ma C, Li X, Tao D, Gong J and Qin J: The CEA-/lo colorectal cancer cell population harbors cancer stem cells and metastatic cells. Oncotarget 7: 80700-80715, 2016

32. Lee YJ, Lee HR, Nam CM, Hwang UK and Jee SH: White blood cell count and the risk of colon cancer. Yonsei Med J 47: 646-656, 2006

33. Fares J, Fares MY, Khachfe HH, Salhab HA and Fares Y: Molecular principles of metastasis: A hallmark of cancer revisited. Signal Transduct Target Ther 5: 28, 2020.

34. Saito G, Sadahiro S, Kamata H, Miyakita H, Okada K, Tanaka A and Suzuki T: Monitoring of serum carcinoembryonic antigen levels after curative resection of colon cancer: Cutoff values determined according to preoperative levels enhance the diagnostic accuracy for recurrence. Oncology 92: 276-282, 2017.
35. Doll D, Keller L, Maak M, Boulesteix AL, Siewert JR, Holzmann B and Janssen KP: Differential expression of the chemokines GRO-2, GRO-3, and interleukin-8 in colon cancer and their impact on metastatic disease and survival. Int $\mathrm{J}$ Colorectal Dis 25: 573-581, 2010.

36. Nguyen HT and Duong HQ: The molecular characteristics of colorectal cancer: Implications for diagnosis and therapy. Oncol Lett 16: 9-18, 2018

37. Ankathil R, Mustapha MA, Abdul Aziz AA, Mohd Shahpudin SN, Zakaria AD, Abu Hassan MR and Musa KI: Contribution of genetic polymorphisms of inflammation response genes on sporadic colorectal cancer predisposition risk in Malaysian patients-a case control study. Asian Pac J Cancer Prev 20: $1621-1632,2019$

38. Huang S, Mills L, Mian B, Tellez C, McCarty M, Yang XD, Gudas JM and Bar-Eli M: Fully humanized neutralizing antibodies to interleukin-8 (ABX-IL8) inhibit angiogenesis, tumor growth, and metastasis of human melanoma. Am J Pathol 161: $125-134,2002$

39. Yoshimoto T, Chiba Y, Furusawa J, Xu M, Tsunoda R, Higuchi K and Mizoguchi I: Potential clinical application of interleukin-27 as an antitumor agent. Cancer Sci 106: 1103-1110, 2015.

40. Oniki S, Nagai H, Horikawa T, Furukawa J, Belladonna ML, Yoshimoto T, Hara I and Nishigori C: Interleukin-23 and interleukin-27 exert quite different antitumor and vaccine effects on poorly immunogenic melanoma. Cancer Res 66: 6395-6404, 2006.

41. Kourko O, Seaver K, Odoardi N, Basta S and Gee K: IL-27, IL-30, and IL-35: A cytokine triumvirate in cancer. Front Oncol 9: 969, 2019.

42. Fabbi M, Carbotti G and Ferrini S: Dual roles of IL-27 in cance biology and immunotherapy. Mediators Inflamm 2017: 3958069, 2017.

43. Park YJ, Ryu H, Choi G, Kim BS, Hwang ES, Kim HS and Chung Y: IL-27 confers a protumorigenic activity of regulatory T cells via CD39. Proc Natl Acad Sci USA 116: 3106-3111, 2019.

44. Diakowska D, Lewandowski A, Markocka-Mączka K and Grabowski K: Concentration of serum interleukin-27 increase in patients with lymph node metastatic gastroesophageal cancer. Adv Clin Exp Med 22: 683-691, 2013.

45. Lu D, Zhou X, Yao L, Liu C, Jin F and Wu Y: Clinical implications of the interleukin 27 serum level in breast cancer. J Investig Med 62: 627-631, 2014.

46. Di Carlo E, Sorrentino C, Zorzoli A, Di Meo S, Tupone MG, Ognio E, Mincione G and Airoldi I: The antitumor potential of Interleukin-27 in prostate cancer. Oncotarget 5: 10332-10341, 2014.

47. Izawa N, Shitara K, Masuishi T, Denda T, Yamazaki K, Moriwaki T, Okuda H, Kondoh C, Nishina T, Makiyama A, et al: Vascular endothelial growth factor (VEGF)-D and clinical outcomes in metastatic colorectal cancer (mCRC) patients (pts) treated with second-line FOLFIRI plus bevacizumab (Bev): A biomarker study of the WJOG 6210G trial. J Clin Oncol 38 (Suppl 4): S226, 2020

48. Cao D, Hou M, Guan YS, Jiang M, Yang Y and Gou HF: Expression of HIF-1alpha and VEGF in colorectal cancer: Association with clinical outcomes and prognostic implications. BMC Cancer 9: 432, 2009.

49. Raluca BA, Cimpean AM, Cioca A, Cretu O, Mederle O, Ciolofan A, Gaje P and Raica M: Endothelial cell proliferation and vascular endothelial growth factor expression in primary colorectal cancer and corresponding liver metastases. Asian Pac J Cancer Prev 16: 4549-4553, 2015.

50. Jubb AM, Hurwitz HI, Bai W, Holmgren EB, Tobin P, Guerrero AS, Kabbinavar F, Holden SN, Novotny WF, Frantz GD, et al: Impact of vascular endothelial growth factor-a expression, thrombospondin-2 expression, and microvessel density on the treatment effect of bevacizumab in metastatic colorectal cancer. J Clin Oncol 24: 217-227, 2006.

51. Fiore A, Carraresi L, Morabito A, Polvani S, Fortunato A, Lastraioli E, Femia AP, De Lorenzo E, Caderni G and Arcangeli A: Characterization of hERG1 channel role in mouse colorectal carcinogenesis. Cancer Med 2: 583-594, 2013.

This work is licensed under a Creative Commons Attribution-NonCommercial-NoDerivatives 4.0 International (CC BY-NC-ND 4.0) License. 